\title{
Dual Network Model for Red Blood Cell Membranes
}

\author{
David H. Boal and Udo Seifert \\ Department of Physics, Simon Fraser University, Burnaby, British Columbia, Canada V5A IS6
}

\author{
Andreas Zilker \\ Department of Pathology, University of British Columbia, Vancouver, British Columbia, Canada V6T 2 B5
}

(Received 3 August 1992)

\begin{abstract}
A two-component network is studied by Monte Carlo simulation to model the lipid/spectrin membrane of red blood cells. The model predicts that the shear modulus decreases rapidly with the maximum length of the model spectrin and should be in the $10^{-7} \mathrm{~J} / \mathrm{m}^{2}$ range for human red blood cells. A simplified model for the isolated spectrin network shows a negative Lamé coefficient $\lambda$. Transverse fluctuations of the dual membrane are found to be fluidlike over the range of wavelengths investigated.
\end{abstract}

PACS numbers: $87.22 . \mathrm{Bt}, 05.40 .+\mathrm{j}, 68.10 .-\mathrm{m}, 82.70 .-\mathrm{y}$

Erythrocytes are remarkable elastic bodies [1]. They are stiff enough to recover their biconcave equilibrium shape after being squeezed through narrow capillaries only $\frac{1}{3}$ of their diameter. Yet they are soft enough to allow for thermally excited shape fluctuations as seen in the flicker phenomenon [2]. Their basic membrane architecture is essentially a three-component system. The lipid bilayer provides a relatively large area compression modulus and high flexibility for bending deformations. The cytoskeleton on the cytoplasmatic side of this bilayer consists mainly of spectrin tetramers linked together at junctional complexes to form a quasihexagonal network. The spectrin network and its junctional complexes are attached to the bilayer by integral membrane proteins. The third component, the glycocalix, controls the interaction with the extracellular matrix or other cells.

Understanding how the mechanical properties of the red blood cell (RBC) membrane arise from its structural composition remains a significant challenge. While equilibrium shapes, shape transformations, and fluctuations of giant lipid bilayer vesicles are now understood on the basis of continuum elastic models for the bending energy [3], it is not yet fully clear whether and how the cytoskeleton affects the equilibrium shape of the erythrocyte and its fluctuations. Recently, analysis of the flicker spectrum revealed a wavelength dependence characteristic of fluid membranes and, thus, no effect of the spectrin network for wavelengths less than $1.5 \mu \mathrm{m}$ [4]. Likewise, direct measurement of the mean-square thickness fluctuations [5], which are dominated by the long-wavelength shape fluctuations, seems to suggest that the shear modulus for small fluctuations is much less than the one obtained from the micromechanical experiments [6]. A possible explanation for such a discrepancy might be a nonlinear behavior of the network with respect to shear distortions. If the spectrin tethers can be expanded to a certain length with almost no cost in energy, then one might expect that small fluctuations basically do not involve the network, whereas larger distortions as measured by micromechanical experiments involve shear of this network. These effects are difficult to model within a continuum mechanical model.

In this paper, we present a model for RBC membranes which draws its inspiration from computer simulations developed for both polymerized [7,8] and fluid [9] membranes. We capture what we believe to be the two essential aspects of the RBC membrane, namely, the fluid bilayer and the cytoskeleton. In our model, the elasticity is of entropic origin, an aspect previously incorporated in phenomenological continuum models $[10,11]$. Using Monte Carlo simulations, we first determine the twodimensional elastic constants of the model membrane and then investigate its out-of-plane fluctuations in three dimensions.

We define the membrane as a two-dimensional surface represented by a fixed number $N$ of hard spherical beads (or vertices) of diameter $a$. Two different sets of straight flexible tethers connect the beads together. One set of connections are fluid tethers, shown in light grey in Fig. 1 , whose maximal length of $\sqrt{3} a$ enforces membrane self-avoidance. These tethers are fluid in that they can migrate from vertex to vertex. Every bead has an average of six fluid tethers attached to it. A second set of connections are what we call spectrin tethers, which form a hexagonal network with fixed connectivity, as shown in white in Fig. 1. The spectrin tethers have a maximal length of $s_{\max }$, which is the main model parameter. One out of every 36 beads is an anchor point at which a hexagonal junction point of the spectrin network is attached to the fluid network. Spectrin tethers are allowed to intersect, as they only represent the in-plane projections of the three-dimensional protein chains. Beads connected only by fluid tethers move freely through the membrane like lipid molecules. Anchor beads have their movement restricted by the spectrin tether constraints, as do the junction complexes in RBC's. However, the beads are used only to mathematically represent the membrane, and their number density should not be equated with the number density of lipid molecules.

In the first set of simulations to determine the elastic moduli, we confine the vertex positions to a twodimensional plane. We use a Metropolis Monte Carlo 


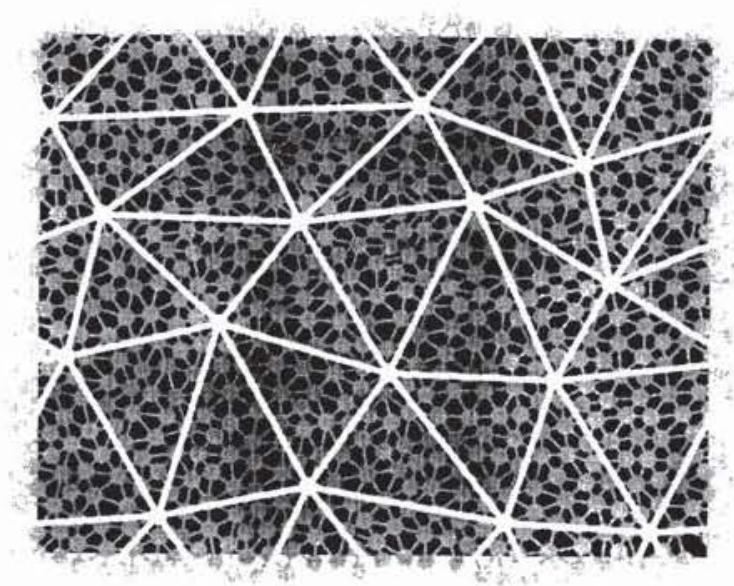

FIG. 1. Sample configuration for $s_{\max }=9$ viewed perpendicular to the $x-y$ plane. The fluid tethers are grey, while the spectrin tethers are white.

technique to generate a set of appropriately weighted sample configurations. A sweep across the membrane involves the following steps: (i) An attempt is made to change the position of each vertex by choosing a new position randomly from within a square box of length $2 l$ to the side centered on the old position, where we choose $l=0.1$ a. (ii) An attempt is made to reconnect every fluid tether following the procedure of Baumgartner and Ho [9]. In this procedure, a tether is removed and replaced with a new tether connecting the two "opposite" vertices which (along with the vertices at the ends of the original tether) define the two triangles having the original tether in common. The spectrin tethers are not subject to procedure (ii) since their attachment is permanent. Each trial move is accepted if it does not violate the tether length and bead size constraints.

A rectangular membrane "patch" subject to periodic boundary conditions in the $x$ and $y$ directions is used in the simulation. An isobaric simulation is performed by allowing the rectangle lengths $L_{x}$ and $L_{y}$ to vary independently. There is one trial move to rescale the rectangle size per sweep. The rescaling moves are accepted with a pseudo Boltzmann factor [12]

$$
W=\exp [-\beta P \Delta A+N \ln (1+\Delta A / A)],
$$

where $P$ is the pressure and $\Delta A$ is the difference in the area $\left(A \equiv L_{x} L_{y}\right)$ before and after the rescaling. Except where otherwise noted, the pressure has been set to zero.

At least 100 or 200 sample configurations are generated at each parameter set. Each configuration is separated by a "Rouse time" $\tau \equiv N / l^{2}$ Monte Carlo sweeps. The equilibration time is within a factor of 3 or less of the Rouse time for membranes of the size used here $[7,8,13]$, so each initialization is allowed to relax for $10 \tau$ before sample collection commences. Between $5 \times 10^{6}$ and $10 \times 10^{6}$ attempted moves are made on each vertex and tether for membranes with $N=576$. The entire simula-

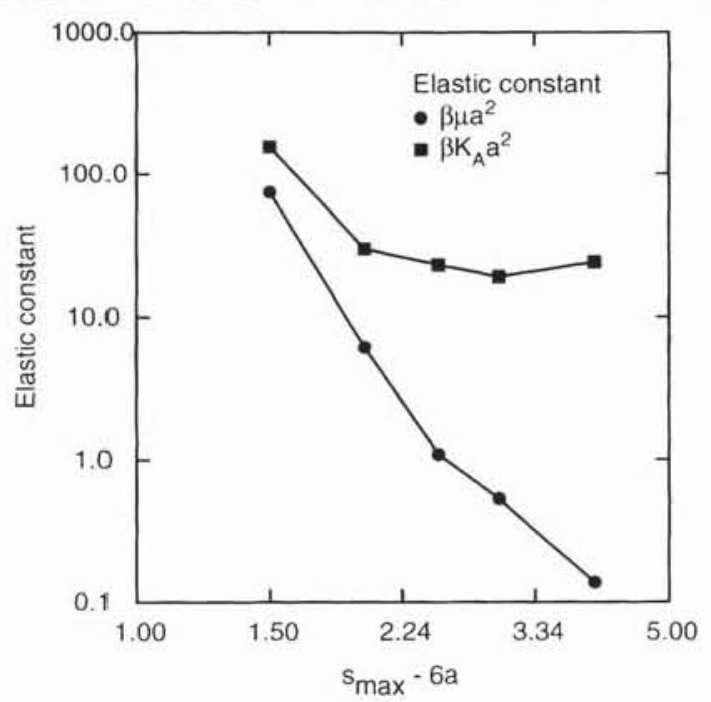

FIG. 2. Logarithmic plot of the elastic constants $\beta \mu a^{2}$ (circles) and $\beta K_{A} a^{2}$ (squares) as a function of the maximum spectrin length $s_{\max }$ in the dual network. Our estimated uncertainties are in the $10 \%$ range.

tion required approximately ten CPU months on a MIPS R3000 processor.

Our flat membrane is described by two lateral displacement fields $u_{1}$ and $u_{2}$ which are functions of the reference coordinate system $\left(x_{1}, x_{2}\right)$. In terms of the strain tensor $u_{i j}=\left(\partial u_{i} / \partial x_{j}+\partial u_{j} / \partial x_{i}\right) / 2$, the elastic energy is [14]

$$
\begin{aligned}
H_{\mathrm{el}}=\int d^{2} x & \left\{K_{A}\left(u_{11}+u_{22}\right)^{2} / 2\right. \\
& \left.+\mu\left[\left(u_{11}-u_{22}\right)^{2} / 2+2 u_{12}^{2}\right]\right\},
\end{aligned}
$$

where $K_{A}$ and $\mu$ are the area compressibility modulus and shear modulus, respectively. Computationally, $K_{A}$ and the Young's moduli $Y_{x}$ and $Y_{y}$ are determined from fluctuations of $L_{x}, L_{y}$, and $A$ :

$$
\begin{aligned}
& \beta K_{A}=\langle A\rangle /\left(\left\langle A^{2}\right\rangle-\langle A\rangle^{2}\right), \\
& \beta Y_{x}=\left[\langle A\rangle\left(\left\langle L_{x}^{2}\right\rangle /\left\langle L_{x}\right\rangle^{2}-1\right)\right]^{-1}, \\
& \beta Y_{y}=\left[\langle A\rangle\left(\left\langle L_{y}^{2}\right\rangle /\left\langle L_{y}\right\rangle^{2}-1\right)\right]^{-1} .
\end{aligned}
$$

Finally, the shear modulus $\mu$ can be obtained from $Y$ and $K_{A}$ via

$$
\mu=Y K_{A} /\left(4 K_{A}-Y\right),
$$

where we use the average of $Y_{x}$ and $Y_{y}$ for $Y$ in Eq. (6).

The behavior of these constants for the model network is shown in Fig. 2. The smallest value for $s_{\max }$ consistent with our density of anchor vertices is $6 a$, where all the beads touch at their hard-core limit. Such a network should have infinitely large $\mu$ and $K_{A}$. From Fig. 2 we see that $\mu$ and $K_{A}$ decrease from large values as $s_{\max }$ increases from $6 a$. The compression modulus $\beta K_{A} a^{2}$ decreases to about 20 and remains near this value as $s_{\max }$ becomes large. The shear modulus, on the other hand, 
falls approximately as $s_{\max }^{-5 \pm 1}$ over the larger values of $s_{\max }$ studied. This behavior is expected since networks with larger $s_{\max }$ can support larger shear deformations.

The compression modulus at large $s_{\max }$ can be understood by considering the single-component fluid [9] or polymerized [7,8] network model. Kantor and Nelson [8] point out that in a two-dimensional network of harmonic springs $K_{A}=2 \mu=3^{1 / 2} k / 2$, where $k$ is the spring constant. Kantor and Nelson estimate that the square-well tethers correspond to $\beta k a^{2} \approx 22$ and hence they predict $\beta K_{A} a^{2} \approx 20$. In a separate simulation of two-dimensional networks, we obtain $\beta K_{A} a^{2}=15 \pm 2$ for a polymerized network and $\beta K_{A} a^{2}=18 \pm 2$ for a pure fluid network. These results are in the range expected from the harmonic network and are similar to the value of the dual net. Thus, the compression modulus of the dual net at large $s_{\max }$ is determined by its fluid component and not its spectrin component.

To understand the shear modulus at large $s_{\max }$ we simulate a pure two-dimensional spectrin network with fixed hexagonal connectivity, $N_{j}$ vertices, and a tether length ranging from 0 to $s_{\max }$. The network is subject to a tension (or negative pressure) $P<0$ so that the only independent variable in the simulation is $\beta P s_{\max }^{2}$. The tension is used to control the area of the membrane so the pure spectrin network can be compared with the dual network. The simulation is performed using the computational rules set out above for the spectrin part of the dual network.

The shear modulus $\beta \mu s_{\max }^{2}$ is shown in Fig. 3 as a func-

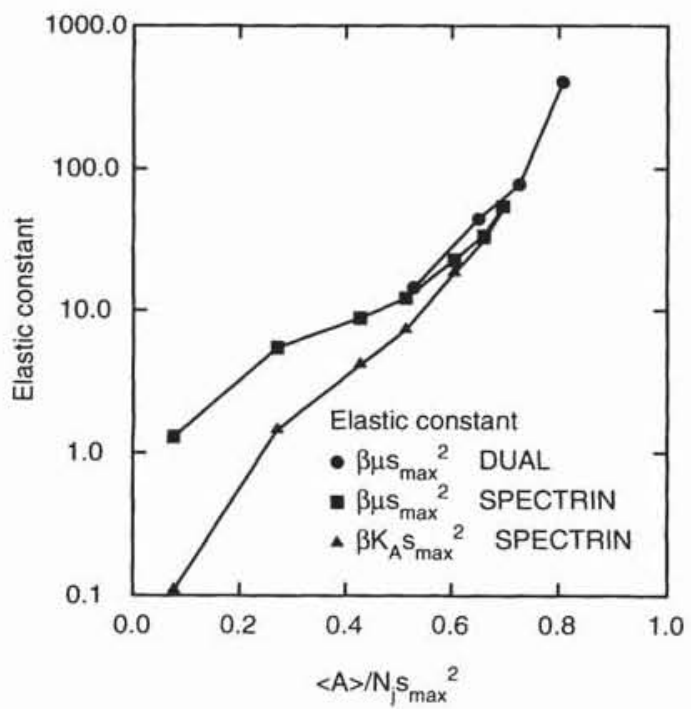

FIG. 3. Comparison of the shear modulus $\beta \mu s_{\max }^{2}$ for the dual network (circles) and the pure spectrin network (squares) as a function of the area per junction vertex $\langle A\rangle / N_{j} s_{\max }^{2}$. The compression modulus $\beta K_{A} s_{\max }^{2}$ of the pure spectrin network is also shown (triangles). Most pure spectrin data were calculated with $N_{j}=144$, except the two smallest areas which used $N_{j}=576$. tion of the area per junction vertex $\langle A\rangle / N_{j} s_{\max }^{2}$ for the dual network and the pure spectrin network. To within our $10 \%$ uncertainties, both networks have similar values of $\beta \mu s_{\max }^{2}$ for those values of $\langle A\rangle / N_{j} s_{\max }^{2}$ which correspond to large $s_{\max }$ in the dual model. Hence, we conclude that the shear modulus at large $s_{\max }$ is determined by the spectrin network. Also shown in Fig. 3 is the compression modulus $\beta K_{A} s_{\max }^{2}$ of the spectrin network. The fact that $K_{A}$ is less than $\mu$ indicates that the second Lamé coefficient [14] $\lambda=K_{A}-\mu$ is negative. Materials with negative $\lambda$ expand transversely when stretched longitudinally.

We can use Fig. 3 to make a "first-principles" estimate of the shear modulus of human RBC's [15], for which the spectrin has a maximal extension $\left(s_{\max }\right)$ of $\approx 200 \mathrm{~nm}$ and an average extension of $\approx 75 \mathrm{~nm}$ [1]. This corresponds to $\langle A\rangle / N_{j} s_{\max }^{2} \approx 0.12$, for which we obtain $\beta \mu s_{\text {max }}^{2} \approx 2$ from Fig. 3. This value is equivalent to $\mu \approx 2 \times 10^{-7} \mathrm{~J} / \mathrm{m}^{2}$ and is more than an order of magnitude less than what is found in the micropipette experiments $\left[\mu=(6-9) \times 10^{-6} \mathrm{~J} / \mathrm{m}^{2}[6,16]\right]$. Inclusion of outof-plane fluctuations and protein steric effects will undoubtedly affect the numerical value of this prediction. At face value, the prediction supports the hypothesis that the shear modulus determined by fluctuations $[4,5]$ is considerably smaller than that determined by micromechanical experiments [6].

We now investigate the out-of-plane fluctuations of the dual network by embedding the membrane in three dimensions. To incorporate out-of-plane bending resistance, we add to our simulation a discrete version $[7,8]$ of the continuum Helfrich Hamiltonian [17]

$$
\beta H=\kappa_{\text {dis }} \sum\left(1-\mathbf{n}_{i} \cdot \mathbf{n}_{j}\right),
$$

where each $\mathbf{n}$ is a unit vector normal to the plane formed by three vertices which are all nearest neighbors to one another. The sum is over all n's whose defining plaquettes share a common tether.

We use the same Metropolis algorithm described above, modified (i) by using a spatial cube for each trial vertex move and (ii) by accepting or rejecting a move according to the Boltzmann weight $\exp (-\beta \Delta H)$, where $\Delta H$ is the energy difference of the trial move as determined by Eq. (7). For low enough temperature, i.e., $\kappa_{\text {dis }} \gg 1$, the out-of-plane fluctuations are moderate and a unique height function $h(\mathbf{x})$ can be defined for every vertex position $\mathbf{x}$ in the $x-y$ plane. Fluctuations are characterized by the correlation function

$$
C(\mathbf{q}) \equiv\left\langle|u(\mathbf{q})|^{2}\right\rangle
$$

of the Fourier-transformed height $u(\mathbf{q}) \equiv S^{-1} \int d^{2} x h(\mathbf{x})$ $\times \exp (i q \cdot \mathbf{x})$, where $S$ is the surface area over which the two-dimensional integral $d^{2} x$ is performed. For long wavelengths or small $q, C(q) \approx q^{-(2+2 \zeta)}$, where the roughness exponent $\zeta$ determines how fluctuations grow with spatial separation, that is, $\left\langle[h(\mathbf{x})-h(0)]^{2}\right\rangle \approx x^{2 \zeta}$ 
for large $x$. Fluid membranes are characterized by $\zeta=1$, while simulations variously show $\zeta \approx 0.65$ for tethered membranes $[13,18-20]$ and $\zeta=\frac{1}{2}$ in a continuum model of a polymerized membrane [21] and in a perturbation theory calculation [22].

For the simulation we choose $s_{\max }=8$, which is a value in the crossover regime between the harmonic ( $s_{\max }$ $\approx 6-7$ ) and pure fluid (large $s_{\max }$ ) networks. Over our available range of $q a$ from about 0.2 to 2 , the in-plane correlation function drops by 3 orders of magnitude and scales like $q^{-4.0 \pm 0.1}$ characteristic of pure fluid membranes (for which we independently find $q^{-3.9 \pm 0.1}$ ). The present system size is too small to show the predicted crossover to a "solid" or "tetherlike" behavior of fluctuations at larger length scales $[21,22]$. For a pure polymerized network, we find $C(q)$ scales like $q^{-3.7 \pm 0.2}$ for $N=576$, indicating that we require larger system sizes to reach the asymptotic regime [18].

In conclusion, we introduce a dual network model for the $\mathrm{RBC}$ membrane in which the maximum spectrin length $s_{\max }$ is the essential parameter. In two dimensions, the mechanical properties of this network are determined entirely by entropy and geometry. At large $s_{\max }$ the elastic moduli decouple: The compression modulus is a function of the fluid bilayer properties while the shear modulus falls rapidly with the spectrin length. A simplified model for the spectrin network shows a negative Lamé coefficient $\lambda$. We predict the shear modulus for human RBC's to be in the range $(1-3) \times 10^{-7} \mathrm{~J} / \mathrm{m}^{2}$. In three dimensions, the transverse fluctuations are fluidlike over the range of wavelengths available in the simulation.

This work is supported in part by the Natural Sciences and Engineering Research Council of Canada, the Canadian Institute for Advanced Research, and the Alexander von Humboldt Foundation. We thank E. Evans, R. Grebe, M. Narla, E. Sackmann, and M. Zuckermann for many stimulating conversations.

[1] For a review, see T. L. Steck, in Cell Shape: Determinants, Regulation and Regulatory Role, edited by W. Stein and F. Bronner (Academic, New York, 1989), Chap. 8.
[2] F. Brochard and J. F. Lennon, J. Phys. (Paris) 36, 1035 (1975).

[3] For a review, see R. Lipowsky, Nature (London) 349, 475 (1991).

[4] A. Zilker, M. Ziegler, and E. Sackmann, Phys. Rev. A (to be published).

[5] M. A. Peterson, H. Strey, and E. Sackmann, J. Phys. II (France) 2, 1273 (1992).

[6] R. Waugh and E. Evans, Biophys. J. 35, 637 (1979).

[7] Y. Kantor, M. Kardar, and D. R. Nelson, Phys. Rev. Lett. 57, 791 (1986).

[8] Y. Kantor and D. R. Nelson, Phys. Rev. A 36, 4020 (1987); see also Y. Kantor, Phys. Rev. A 39, 6582 (1989)

[9] A. Baumgartner and J.-S. Ho, Phys. Rev, A 41, 5747 (1990).

[10] T. Stokke, A. Mikkelsen, and A. Elgsaeter, Eur. Biophys. J. 13, 203 (1986); 13, 219 (1986).

[11] E. A. Evans and R. Skalak, Mechanics and Thermodynamics of Biomembranes (CRC Press, Boca Raton, 1980).

[12] W. W. Wood, J. Chem. Phys. 48, 415 (1968); see also J. P. Hansen and 1. R. McDonald, Theory of Simple Liquids (Oxford Univ. Press, New York, 1986).

[13] M. Plischke and D. Boal, Phys. Rev. A 38, 4943 (1988); D. Boal, E. Levinson, D. Liu, and M. Plischke, Phys. Rev. A 40, 3292 (1989).

[14] See, for example, L. D. Landau and E. M. Lifshitz, Theory of Elasticity (Pergamon, London, 1959).

[15] For a scaling argument to estimate $\mu$ within continuum theories, see Refs. [10,11].

[16] R. M. Hochmuth, in Handbook of Bioengineering, edited by R. Skalak and S. Chien (McGraw-Hill, New York, 1987), pp. 12.1-12.17; J. Engelhardt and E. Sackmann, Biophys. J. 54, 495 (1988)

[17] W. Helfrich, Z. Naturforsch. 28C, 693 (1973); H. J. Deuling and W. Helfrich, J. Phys. (Paris) 37, 1335 (1976).

[18] F. Abraham and D. R. Nelson, J. Phys. (Paris) 51, 2653 (1990).

[19] S. Leibler and A. Maggs, Phys. Rev. Lett. 63, 406 (1989).

[20] J.-S. Ho and A. Baumgartner, Europhys. Lett. 12, 295 (1990).

[21] R. Lipowsky and M. Girardet, Phys. Rev. Lett. 65, 2893 (1990).

[22] D. R. Nelson and L. Peliti, J. Phys. (Paris) 48, 1085 (1987). 


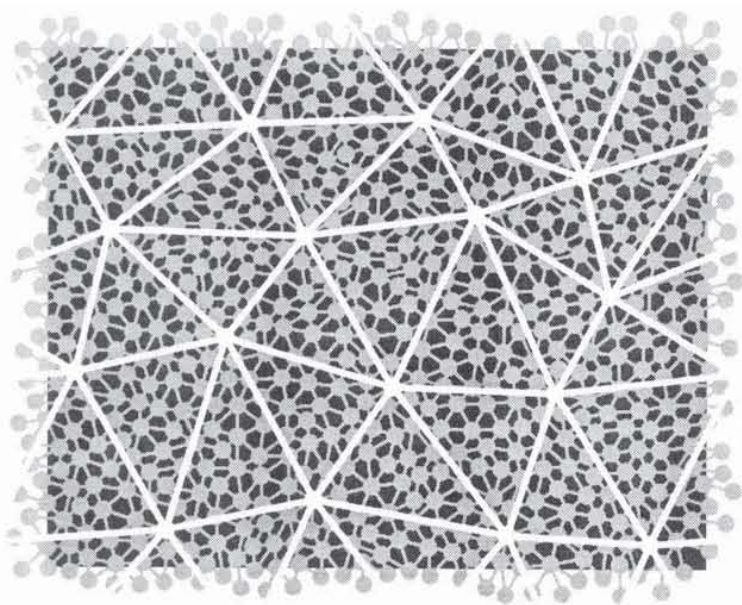

FIG. 1. Sample configuration for $s_{\max }=9$ viewed perpendicular to the $x-y$ plane. The fluid tethers are grey, while the spectrin tethers are white. 